\title{
Conditional Equilibrium and the Equivalence of Microcanonical and Grandcanonical Ensembles in the Thermodynamic Limit
}

\author{
Michael Aizenman ${ }^{1 \star}$, Sheldon Goldstein ${ }^{2 \star \star}$, and Joel L. Lebowitz ${ }^{2 \star \star \star}$ \\ 1 Department of Physics, Princeton University, Princeton, New Jersey 08540, USA \\ 2 Department of Mathematics, Rutgers University, New Brunswick, New Jersey 08903, USA
}

\begin{abstract}
Equivalence (allowing for convex combinations) of microcanonical, canonical and grandcanonical ensembles for states of classical systems is established under very mild assumptions on the limiting state. We introduce the notion of conditional equilibrium (C.E.), a property of states of infinite systems which characterizes convex combinations of limits of microcanonical ensembles. It is shown that C.E. states are, under quite general conditions, mixtures of Gibbs states.
\end{abstract}

\section{Introduction}

Systems of infinite spatial extent [1-3] offer mathematically convenient idealizations of macroscopic equilibrium systems. The statistical mechanical theory of such systems may be obtained either by considering the thermodynamic (infinite volume) limit of finite systems described by appropriate Gibbs ensembles (e.g., micro-canonical, canonical, grand-canonical, pressure) or by considering equilibrium states of infinite systems directly. While the first route is the more physical, the latter is mathematically more direct and can often provide useful insights into the phenomena for which the large size (on a molecular scale) of macroscopic systems plays an essential role, e.g., phase-transitions. In addition the formal theory of infinite systems may offer useful mathematical tools for the study of local phenomena in macroscopic systems. Various results valid in the thermodynamic limit can be formulated as simple properties of the infinite system. It is for these reasons that the infinite system point of view is often adopted.

In the infinite system formalism, equilibrium macroscopic systems are commonly represented by Gibbs states, or measures, on the space of locally finite configurations $[2,3]$. These are defined by the DLR equations $[2,3]$ which are satisfied by the limits of finite volume grand-canonical ensembles. One may also define "conditional equilibrium" states (C.E.) by similar equations which are

* $\quad$ Supported in part by NSF Grant No. MCS 75-21684 A02

$\star \star$ Supported in part by NSF Grant No. MPS 72-04534

$\star \star \star$ Supported in part by NSF Grant No. Phy 77-22302 
satisfied by the limits of the corresponding canonical and micro-canonical finite volume ensembles. The question naturally arises as to the relationship between C.E. and Gibbs states; it was a fundamental belief of the founders of statistical mechanics that in the thermodynamic limit the various ensembles yield the same results.

The equivalence of different ensembles in this limit can be readily verified as far as thermodynamic properties are concerned [1]. The problem becomes more difficult however when equivalence between correlation functions, i.e., measures, is desired. One difficulty arises from the fact that the existence of a unique limit of the correlations is only assured, in general, at low values of the fugacity (and also at high temperatures for some class of systems [1]). Indeed for low fugacities a proof of the equivalence of canonical and grand-canonical correlation functions was given by Bogoluibov in 1946 [4].

It may however by expected that even in the absence of a unique limit state, as would happen at phase transitions where several phases can coexist, there should still be an equivalence between the "sets" of states corresponding to limits of different ensembles or the different characterizations of infinite volume states. This indeed was proven by Thompson [5] for lattice systems; cf. also Georgii [6] and Martin-Löf [7]. For continuous systems Georgii [8] proved the equivalence of canonical Gibbs states and Gibbs states under certain assumptions. It is the purpose of this paper to prove the equivalence between Gibbs states and a general class of C.E. states, of which limits of canonical and microcanonical ensembles are special cases, for classical systems with realistic Hamiltonians.

Our interest in this problem grew out of our work on the stability of stationary (time invariant) measures of infinite classical systems [9]. It was shown there, following the work of Haag et al. [10] on quantum states, that under certain conditions stable stationary states satisfy a classical KMS condition. That condition was shown by Gallavotti and Verboven [11], under restrictive conditions equivalent to low fugacities, to imply that these states are Gibbs states. Later the KMS condition was shown [12] to be equivalent to canonical states which, by Georgii's result, shows its equivalence to Gibbs states quite generally. While we do not know of any dynamical condition which leads to the more general class of C.E. states considered here, they do seem to be natural generalizations of Gibbs states. Similar questions have been independently considered by Preston [13] who obtained partial results in this direction.

The general formalism and notation are introduced in Parts a) and b) of Section 2. In 2c) we introduce the C.E. states and discuss their connection with limits of microcanonical ensembles (Theorem 2.1 and Remark 2.2). The main problem, stated in $2 \mathrm{~d}$ ), is the relation of C.E. states to Gibbs states. This problem may be simply described in terms of an entropy function which is introduced in Section 3. We study the C.E. states by first decomposing them to extremal C.E. states which are the subject of Section 4. The equivalence of the C.E. to DLR condition is obtained there (Theorems 4.2 and 4.3) under certain regularity assumptions. While one is usually willing to assume some regularity of states of interest, the regularity of the extremal components of a given state is already a strong statement, as shown by Example 4.1. The discussion in Section 4 is quite general, applying both to continuous and to lattice systems and to states which are C.E. with respect to 
collections of extensive variables. In Section 5 we restrict our attention to continuous systems whose Hamiltonians contain kinetic terms. It is shown, using a criterion (Lemma 5.1) derived from the results of Section 4, that a C.E. state is, under very mild additional assumptions, a combination of Gibbs states (Theorem 5.1). In Section 6 we apply the method and results of Section 4 to obtain quite general results about canonical states.

\section{Setup and Statement of the Problem}

\section{a) Phase Space}

Let $\Gamma=\mathbb{R}^{d} \times \mathscr{X}$ be the one particle phase space (position space $\times$ space of the other degrees of freedom) of a classical system of particles, equipped with the measure $v_{1}$.

Example 1.1. Five cases of interest are:

1) $\mathscr{X}=\mathbb{R}^{d}$, describing the momentum degrees of freedom, $d v_{1}=d^{d} q d^{d} p$.

2) $\mathscr{X}=\{1, \ldots, n\}$, for $n$ types of particles, $d v_{1}=d^{d} q \times$ counting measure.

3) $\mathscr{X}=\mathbb{R}^{d} \times\{1, \ldots, n\}$.

4) $\mathscr{X}=\{1, \ldots, n\}, d v_{1}=\sum_{i \in \mathbb{Z}^{d}} \delta\left(d^{d} q-i\right) \times$ counting measure, describing a system on a lattice.

5) $\mathscr{X}=\mathbb{R}, d v_{1}=\sum_{i \in \mathbb{Z}^{d}} \delta\left(d^{d} q-i\right) \times d s$ describing a lattice of continuous spins.

We assume that $\mathscr{X}$ is a complete separable metric space.

Let $\Lambda$ refer to bounded open subsets of $\mathbb{R}^{d}$. We shall also denote, with a slight abuse of notation, $\Lambda \times \mathscr{X} \subset \Gamma$ by $\Lambda$. We write $\Lambda^{c}=\mathbb{R}^{d} \backslash \Lambda$ (or $\Gamma \backslash \Lambda$ ).

Definition 2.1. A configuration, $\omega$, is the equivalence class, under permutations, of a (possibly finite) sequence $\left\{\omega_{j}\right\}$ with $\omega_{j} \in \Gamma \forall j$.

We write $\omega_{j}=\left(q_{j}, p_{j}\right), \omega_{j}=\left(q_{j}, \alpha_{j}\right), \omega_{j}=\left(q_{j}, p_{j}, \alpha_{j}\right)$ for cases 1), 2), and 3) of Example 1.1, respectively.

The set theoretic notation may be extended to deal with such equivalence classes (keeping track of multiplicities of coincident particles: $\omega_{i}=\omega_{j}$ for $i \neq j$ ). Thus we will write $\omega=\left\{\omega_{i}\right\}$.

For configurations $\omega, \bar{\omega}$, and $V \subset \Gamma$ we write

$$
\begin{aligned}
\omega \bar{\omega} & =\omega \cup \bar{\omega} \\
\omega_{V} & =\omega \cap V \\
N(\omega) & =\operatorname{card} \cdot \omega
\end{aligned}
$$

and

$$
N_{V}(\omega)=N\left(\omega_{V}\right) .
$$

Definition 2.2. $\Omega$, the phase space of an infinite system, is the set of locally finite configurations. These are the configurations $\omega$ for which $N\left(\omega_{A}\right)<\infty$ for all bounded $\Lambda \subset \mathbb{R}^{d}$.

Observables are represented by functions on $\Omega$.

Definition 2.3. An extensive quantity is a function $X(\cdot \mid \cdot)$ on $\Omega_{F} \times \Omega$ where $\Omega_{F}=\{\omega \in \Omega \mid N(\omega)<\infty\}$, which satisfies

$$
X(\xi \eta \mid \omega)=X(\eta \mid \omega)+X(\xi \mid \eta \omega) .
$$


If $X$ is an extensive quantity we let $X_{\Lambda}(\omega)=X\left(\omega_{\Lambda} \mid \omega_{A^{c}}\right)$.

In $X(\xi \mid \omega)$, $\omega$ should be thought of as defining boundary conditions.

Examples of extensive quantities are:

1) $X(\xi \mid \omega)=N(\xi)$

2) For $\mathscr{X}=\mathbb{R}^{d} \times\{1, \ldots, n\}$ or $\mathscr{X}=\{1, \ldots, n\}, N_{i}(\xi \mid \omega)=\operatorname{card} \xi \cap\{\alpha=i\}$.

3) The energy, for a pair potential $v$,

$E(\xi \mid \omega)=\sum_{i}(2 m)^{-1} p_{i}^{2}+\frac{1}{2} \sum_{i \neq j} v\left(q_{i}, q_{j}\right)+\sum_{i, j} v\left(q_{i}, \bar{q}_{j}\right)$,

where $\xi=\left\{\left(q_{i}, p_{i}\right)\right\}$ and $\omega=\left\{\left(\tilde{q}_{i}, \bar{p}_{i}\right)\right\}$.

4) Sum functions. For any function $f$ on $\Omega_{F}$ we define an extensive quantity

$$
\Sigma_{f}(\xi \mid \omega)=\sum_{\substack{\gamma \in \xi \omega \cap \Omega_{F} \\ \gamma \cap \xi \neq \emptyset}} f(\gamma) .
$$

We write $\Sigma_{f}(\omega) \equiv \Sigma_{f}(\omega \mid \emptyset)$. (Strictly speaking, the functions in the last two examples are defined only on the subset of $\Omega_{F} \times \Omega$ for which the sums are absolutely convergent.)

\section{b) States}

The space $\Omega$ admits a natural topology which is generated by the set of functions

$\left\{\Sigma_{f}(\cdot) \mid f \in C\left(\Gamma^{n}\right)\right.$ for some $n \in \mathbb{Z} \operatorname{supp} f=\left\{\gamma \in \Omega_{F} \mid \gamma_{\Lambda^{c}}=\emptyset\right\}$ for some bounded $\left.\Lambda\right\}$

This topology is compatible with a metric with respect to which $\Omega$ is complete and separable (i.e., a "Polish space").

Definition 2.4. 1) We denote by $\mathscr{B}$ the corresponding Borel $\sigma$-algebra on $\Omega$.

2) For any measurable $V \subset \Gamma$ let

$$
\Omega_{V} \equiv\left\{\omega_{V} \mid \omega \in \Omega\right\}
$$

Let $\mathscr{B}_{V}$ be the $\sigma$-algebra on $\Omega$ which is the inverse image of $\mathscr{B} \cap \Omega_{V}$ under the mapping

$$
\begin{aligned}
& \Omega \rightarrow \Omega_{V} \\
& \omega \mapsto \omega_{V} .
\end{aligned}
$$

We identify $\Omega_{\Lambda}$ with $\bigcup_{N=0}^{\infty} \Lambda_{\text {symm. }}^{N}$. Points in $\Omega_{\Lambda}$ will be denoted by $\eta, \xi$.

3) $F_{\Lambda} \equiv \mathscr{B}_{\Lambda^{c}}$ and $F_{\infty}=\bigcap_{\Lambda} F_{\Lambda}=$ (monotone) $\lim _{\Lambda \uparrow \mathbb{R}^{d}} F_{\Lambda}$. By $\Lambda \uparrow \mathbb{R}^{d}$ we mean that $\Lambda$ increases along a sequence of subsets whose union is $\Gamma$. The sequence does not change throughout this discussion.

4) Let $X=\left\{X^{(k)}\right\}_{k=1}^{m}$ be a finite collection of extensive quantities. We denote by $G_{\Lambda}$ the $\sigma$-algebra generated by $F_{\Lambda}$ and $X_{\Lambda}$, and set $G_{\infty} \equiv \bigcap_{\Lambda} G_{\Lambda}=\lim _{\Lambda \uparrow \mathbb{R}^{d}} G_{A^{2}}$. The dependence of $G_{A}$ on $X$ is supressed in this notation, but will be clear from the context.

In other words, $F_{\Lambda}$ represents all the information about $\omega_{\Lambda^{c}}$, for $\omega \in \Omega$, and $G_{\Lambda}$ represents this and the additional information about the "total value" of the extensive quantities $\boldsymbol{X}(\omega)$. 
Definition 2.5. A state $(\mu)$ is a probability measure on $(\Omega, \mathscr{B})$.

The fact that $\Omega$ is a Polish space guarantees ([14]) the existence of regular conditional probabilities with respect to any $\sigma$-subalgebra of $\mathscr{B}$. The regular conditional probabilities with respect to $F_{\Lambda}, \mu\left(\cdot \mid F_{\Lambda}\right)(\omega)$, will also be denoted by $\mu_{\Lambda}(\cdot \mid \omega)$ (and will be regarded as probability measures on $\left.\Omega_{\Lambda}\right) \cdot \mu_{\Lambda}(\cdot \mid \omega)=\mu_{\Lambda}\left(\cdot \mid \omega_{\Lambda^{c}}\right)$ gives the conditional distribution of the configuration in $\Lambda$ given $\omega_{\Lambda^{c}}$.

For any $\Lambda$ let us denote by $v_{A}$ the Liouville measure (which assigns mass 1 to the set $N=0)$ :

$$
v_{\Lambda}=\bigoplus_{N=0}^{\infty}\left(v_{1}\right)^{\times N} / N \text { ! on } \Omega_{\Lambda}
$$

The $v_{A}$ have the convenient factorization property

$$
v_{\Lambda_{1} \cup \Lambda_{2}}=v_{\Lambda_{1}} \times v_{\Lambda_{2}} \text { for } \Lambda_{1} \cap \Lambda_{2}=\emptyset
$$

if we identify $\Omega_{\Lambda_{1} \cup \Lambda_{2}}$ with $\Omega_{\Lambda_{1}} \times \Omega_{\Lambda_{2}}$.

Definition 2.6. $\mu$ is a regular state if, $\forall \Lambda$ and $\mu$-a.e. $\omega \mu_{\Lambda}(\cdot \mid \omega) \ll v_{\Lambda}(\cdot)\left(\mu_{\Lambda}\right.$ is absolutely continuous with respect to $\left.v_{A}\right)$. $(\mu$-a.e. $\omega$ reads " $\mu$-almost every $\omega . ")$

A special role is played by Gibbs states which we define in a slightly generalized way.

Definition 2.7. A state $\mu$ is a Gibbs state with respect to $\boldsymbol{X}$ if $\boldsymbol{X}\left(\xi \mid \omega_{\Lambda^{c}}\right)$ is well defined for $v_{A}(d \xi) \mu(d \omega)$-a.e. $(\xi, \omega)$ and if, for some $\left\{\lambda_{k}\right\} \equiv \lambda \in \mathbb{R}^{m}$

$$
\mu_{\Lambda}(d \xi \mid \omega)=\exp \left(-\lambda \cdot \boldsymbol{X}\left(\xi \mid \omega_{\Lambda^{c}}\right)\right) v_{\Lambda}(d \xi) / \text { Norm }
$$

for every $\Lambda$.

\section{c) Conditional Equilibrium}

Definition 2.8. For $\omega \in \Omega$ the microcanonical ensemble with respect to the extensive quantities $\boldsymbol{X}$ is the probability measure on $\Omega_{\Lambda}$ given by the weak limit

$$
M_{\Lambda}(\cdot \mid \omega)=\lim _{\varepsilon \rightarrow 0}(\text { Norm })^{-1} I\left[\left|\boldsymbol{X}\left(\xi \mid \omega_{\Lambda^{c}}\right)-\boldsymbol{X}_{\Lambda}(\omega)\right|<\varepsilon\right] v_{\Lambda}(d \xi)
$$

Here $I(c)$ is the characteristic function of the set on which the condition $c$ is satisfied and $\omega$ is assumed to be such that the limit exists.

We are now in a position to introduce the main object of our study.

Definition 2.9. A state $\mu$ is a conditional equilibrium (C.E.) state with respect to $X$ if the conditional probabilities with respect to $G_{A}$ are the microcanonical ensembles:

$$
\mu\left(\cdot \mid G_{\Lambda}\right)(\omega)=M_{\Lambda}(\cdot \mid \omega) \text { for } \mu \text {-a.e. } \omega \text {. }
$$

The criterion for a regular state to be a C.E. state may be equivalently formulated as follows.

Definition 2.9. Let $\mu$ be a regular state such that $\boldsymbol{X}\left(\xi \mid \omega_{\Lambda^{c}}\right)$ is well defined for $v_{\Lambda}(d \xi) \mu(d \omega)$-a.e. $(\xi, \omega) . \mu$ is a C.E. state if for every $\Lambda$

$$
\mu_{\Lambda}(d \xi \mid \omega)=g_{\Lambda}\left(X\left(\xi \mid \omega_{\Lambda^{c}}\right), \omega\right) v_{\Lambda}(d \xi)
$$


for some Borel measurable function $g: \mathbb{R}^{m} \times \Omega \rightarrow \mathbb{R}$, i.e., if the density of $\mu_{\Lambda}(d \xi \mid \omega)$ with respect to $v_{A}(d \xi)$ is given, for $\mu$-a.e. $\omega$, by a function which depends on $\xi$ only through $\boldsymbol{X}\left(\xi \mid \omega_{\Lambda^{c}}\right)$.

Remark 2.1. For simplicity, let us consider potential energies, $V$, resulting from finite range pair interactions given by continuous functions with values in $\mathbb{R} \cup\{\infty\}$ (one side compactification of $\mathbb{R}$ ). As remarked by Lanford [15], Hamiltonians $(H)$ which in addition include the kinetic energy, $\Sigma_{p^{2} / 2 m}$, are regular enough for the microcanonical ensembles with respect to $X=\{H, N\}$ to be well defined. The expectation value of a continuous function $h(q, p)=h(\xi)$ is given by

$$
\int h(\xi) M_{A}(d \xi \mid \omega)=(\text { Norm })^{-1} \int d^{l} q p^{-(l-2)} \int \sigma^{l}(d k) h(q, \bar{p} k),
$$

where $l=N_{\Lambda}(\omega) d, \bar{p}^{2}=\max \left\{0,2 m\left[H_{\Lambda}(\omega)-V(q \mid \omega)\right]\right\}$ and $\sigma^{l}(\cdot)$ is the uniform probability distribution on the unit sphere in $\mathbb{R}^{l}$. The cases $l<2$ should be treated separately.

The same applies, with a trivial modification, to systems with hard cores (which are not automatically included in the above class because of the continuity requirements on $V$ ).

Gibbs states are the limits of grand-canonical ensembles. The conditional equilibrium states and microcanonical ensembles are similarly related:

Theorem 2.1. Let a sequence of microcanonical ensembles as in Remark 1 converge weakly to a regular state $\mu$. Then $\mu$ is a conditional-equilibrium state (with respect to $\{H, N\})$.

Proof. By the consistency of microcanonical ensembles it is enough to show that $\forall \Lambda^{\prime} \exists \Lambda \supset \Lambda^{\prime}$ for which $\mu\left(\cdot \mid G_{\Lambda}\right)(\omega)=M_{\Lambda}(\cdot \mid \omega)$, for $\mu$-a.e. $\omega$. We shall prove this equality for any $\Lambda$ such that

$$
\int \mu(d \omega) N_{\partial \Lambda}(\omega)=0 \text {, }
$$

where $\partial \Lambda$ is the boundary of $\Lambda$. The existence of such $\Lambda \supset \Lambda^{\prime}$ follows from the local finiteness of $\Omega$ (and thus does not depend upon the regularity of $\mu$ ).

Let $f(e, n, \omega)$ be a continuous function on $\mathbb{R} \times \mathbb{Z} \times \Omega$ and let $h \in C\left(\Omega_{\Lambda}\right)$, the bounded continuous functions on $\Omega_{\Lambda} . f\left(H_{\Lambda}(\omega), N_{\Lambda}(\omega), \omega_{\Lambda^{c}}\right) h(\omega)$ is continuous unless $N_{\partial \Lambda}(\omega) \neq 0$. This occurs on a closed set of zero measure (by our choice of $\Lambda$ ). Therefore

$$
\begin{aligned}
& \int \mu(d \omega) f\left(H_{\Lambda}(\omega), N_{\Lambda}(\omega), \omega_{\Lambda^{c}}\right) h(\omega) \\
& =\lim _{n \rightarrow \infty} \int M_{\Lambda_{n}}\left(d \omega \mid \omega_{n}\right) f\left(H_{\Lambda}(\omega), N_{\Lambda}(\omega), \omega_{\Lambda^{c}}\right) h(\omega) .
\end{aligned}
$$

For $n$ large enough $\Lambda_{n} \supset \Lambda$ and we may use the consistency of micro-canonical ensembles to obtain

$$
=\lim _{n \rightarrow \infty} \int M_{\Lambda_{n}}\left(d \omega \mid \omega_{n}\right) f\left(H_{\Lambda}(\omega), N_{\Lambda}(\omega), \omega_{\Lambda^{c}}\right) \int M_{\Lambda}(d \bar{\omega} \mid \omega) h(\bar{\omega}) .
$$

It may be easily seen from the expression in Remark 1 that the above integral over $d \bar{\omega}$ yields a function which is continuous in $\omega$ except on $\left\{\omega \mid N_{2 \Lambda}(\omega) \neq 0\right\} \cup\left\{\omega \mid p_{i}=0\right.$ for some $\left.\left(q_{i}, p_{i}\right) \in \omega\right\}$. This too is a closed set of zero measure, since $\mu$ is regular, which implies that the integrals converge to

$$
\int \mu(d \omega) f\left(H_{\Lambda}(\omega), N_{\Lambda}(\omega), \omega_{\Lambda^{c}}\right) \int M_{\Lambda}(d \bar{\omega} \mid \omega) h(\bar{\omega}) .
$$


Since functions of the form of $f$ generate $G$ and $C\left(\Omega_{A}\right)$ generates $\mathscr{B}_{A}$ we may conclude that

$$
\mu\left(\cdot \mid G_{A}\right)(\omega)=M_{\Lambda}(\cdot \mid \omega) \text { for } \mu \text {-a.e. } \omega .
$$

Remark 2.2. Conversely, any C.E. state is a convex integral of limits of microcanonical ensembles [see Eq. (4.1) and P.1-P.4 in Section 4a].

\section{d) The Problem}

Comparing Definition 2.7 with Definition 2.9 one sees that a Gibbs state is also a conditional equilibrium state for the same extensive quantities. Our purpose is to prove that for infinite systems the converse is also true. In view of Theorem 1 , this result will also prove, under the appropriate assumptions, the equivalence of microcanonical and grand-canonical ensembles in the thermodynamic limit. It should be emphasized that we do not discuss the convergence of microcanonical ensembles but rather prove results about states which would result from converging schemes of this type.

As a technical point, let us remark that the defining property $(2.4)$ is preserved under convex combination of states. Consequently, one may not expect to prove that conditional-equilibrium states are Gibbs states but, at most, that they are convex combinations of such states.

Finally, a word about the terminology. There is a temptation to call conditional-equilibrium states simply microcanonical states. Similar terminology has in fact been used by several authors $([6,8,13])$ for similarly defined canonical states (discussed in Section 6). We do not follow this terminology in order to avoid certain confusion. Microcanonical states are generally familiar for finite systems. They are characterized by two properties: i) the total value of certain extensive variables is sharply defined and ii) the "equidistribution" on the corresponding "shell" (which we termed - conditional equilibrium). In the thermodynamical limit which we consider only the local properties of the state retain directly their sense and, in particular, the first property becomes meaningless. Since a very pronounced aspect of microcanonical states of finite systems is thus lost, we choose not to use this term. Notice also another important difference. In a microcanonical state of a finite system there is "more information" specified than in a grand canonical state. However, the notion of conditional equilibrium is weaker than that of equilibrium (which for finite systems corresponds to grand canonical states).

\section{The Entropy Function}

\section{a) Restatement of the Problem in Terms of the Entropy Function}

In order to see more clearly the "thermodynamic" nature of the argument let us introduce the following entropy function.

Definition 3.1. Let $\mu$ be a regular state. The entropy in $\Lambda$ of the configuration $\omega$ is given by the $\left(\mathscr{B}_{\Lambda} \times F_{A}\right.$ measurable $)$ state dependent function, $S_{A}$, defined by:

$$
\mu_{\Lambda}(d \xi \mid \omega)=e^{-S_{\Lambda}(\xi \mid \omega)} v_{\Lambda}(d \omega) \text {. }
$$


We shall also use the shorter notation $S_{\Lambda}(\omega) \equiv S_{\Lambda}\left(\omega_{\Lambda} \mid \omega\right)$. (In terms of the measures $\tau_{A}$ introduced in Section $4, \exp \left[-S_{\Lambda}\right]=d \mu / d \tau_{\Lambda}$, which displays the measurability of $S_{\Lambda}$.)

Remark 3.1. In this terminology:

i) A regular state is a microcanonical state with respect to $X$ iff, for any $\Lambda$ there exists a Borel $\times F_{A}$ measurable function $\hat{S}_{A}: \mathbb{R}^{n} \times \Omega \rightarrow \mathbb{R}$ such that

$S_{\Lambda}(\omega)=\hat{S}_{\Lambda}\left(X_{\Lambda}(\omega) \mid \omega\right)$.

ii) A state is Gibbs state with respect to $X$ iff, for some constant $\lambda \in R^{n}$ and functions $K_{A}$ :

$S_{\Lambda}(\omega)=\lambda \cdot \boldsymbol{X}_{\Lambda}(\omega)+K_{\Lambda}\left(\omega_{\Lambda^{c}}\right)$.

Our problem is, therefore, to prove that if, for specified boundary conditions, the entropy $S_{\Lambda}(\omega)$ is a function only of the extensive quantities then this function is affine, with coefficients which are constant on the elements of the decomposition of the state into extremal C.E. states.

\section{b) Basic Properties of the Entropy}

Remark 3.2. Extensive quantities satisfy the following "differential" condition:

$$
X_{\bar{A}}\left(\omega^{(1)}\right)-X_{\bar{\Lambda}}\left(\omega^{(2)}\right)=X_{\Lambda}\left(\omega^{(1)}\right)-X_{\Lambda}\left(\omega^{(2)}\right)
$$

whenever $\omega_{\Lambda^{c}}^{(1)}=\omega_{\Lambda^{c}}^{(2)}$ and $\bar{\Lambda} \supset \Lambda$.

Although our entropy is not an extensive quantity (see also a remark at Section 6 ), we show in Lemma 1 that it satisfies the above condition [for "typical" $\left.\left(\omega^{(1)}, \omega^{(2)}\right)\right]$. We shall strongly use this property in proving the main result.

Lemma 3.1 (Consistency Condition). Let $\mu$ be a regular state and $S$ the corresponding entropy function; then for $\mu$-a.e. $\omega$

1) $-\infty<S_{A}<\infty$.

2) for $v_{A^{-a} \text {.e. } \xi}$

$$
S_{\bar{\Lambda}}\left(\xi \omega_{\bar{\Lambda} \backslash \Lambda} \mid \omega\right)=S_{\Lambda}(\xi \mid \omega)+C_{\bar{\Lambda}, \Lambda}\left(\omega_{\Lambda} c\right)
$$

if $\bar{\Lambda} \supset \Lambda$.

Proof. 1) It follows from the definition of $S_{A}$ that the set on which $S_{\Lambda}(\omega)=\infty$, i.e., $e^{-S_{\Lambda}(\omega)}=0$, has zero $\mu$ measure. At the other extreme, $S_{\Lambda}>-\infty$ follows the normalizability of $e^{-S_{\Lambda}}$.

2) Consistency of the measures induced in $\Lambda$ and in $\bar{\Lambda}$ implies

$$
\begin{aligned}
& e^{-S_{\Lambda}(\xi \mid \omega)} v_{\Lambda}(d \xi) \mu\left(d \omega_{\Lambda^{c}}\right) \\
& \quad=e^{-S \bar{\Lambda}(\xi \omega \bar{\Lambda} \backslash \Lambda \mid \omega)} v_{\bar{\Lambda}}\left(d \xi d \omega_{\bar{\Lambda} \backslash \Lambda}\right) \mu\left(d \omega_{\bar{\Lambda}^{c}}\right) \\
& \quad=e^{\left.-S \bar{A}^{(\xi \omega} \omega_{\bar{\Lambda} \backslash \Lambda} \mid \omega\right)} v_{\Lambda}(d \xi) v_{\bar{\Lambda} \backslash \Lambda}\left(d \omega_{\bar{\Lambda} \backslash \Lambda}\right) \mu\left(d \omega_{\bar{\Lambda}^{c}}\right)
\end{aligned}
$$

(where use was made of the factorization $v_{\bar{A}}=v_{A} \times v_{\bar{\Lambda} \backslash A}$ ). Thus, for $v_{A}$-a.e. $\xi$ and $\mu$-a.e. $\omega$

$$
S_{\bar{\Lambda}}\left(\xi \omega_{\bar{\Lambda} \backslash \Lambda} \mid \omega\right)=S_{\Lambda}(\xi \mid \omega)+C_{\bar{\Lambda}, \Lambda}\left(\omega_{\Lambda^{c}}\right),
$$

where $C_{\Lambda, \Lambda}\left(\omega_{\Lambda^{c}}\right)<\infty$. 


\section{Extremal Conditional Equilibrium States}

Our goal is to prove that a conditional equilibrium state of an infinite system is a convex combination of Gibbs states. While the "infinite size" of the system is of course crucial, it is not, by itself, a clearly defined condition (nor a sufficient one if by the size one means just the volume-see Example 4.1). We shall, therefore, first prove the above result under an assumption which contains the relevant information about the "size" of the system. In the next section we verify this assumption for a large class of systems.

Intuitively, we shall reason as follows. By definition, for C.E. states the entropy is a function of the extensive quantities. By locally varying the configuration one may define the increase in entropy which corresponds to a change $\Delta X$ in the extensive quantities. Since changes in the entropy and in the extensive quantities can be consistently determined using any large volume, they should be measurable with respect to $G_{\infty}$. Here the distinction between $G_{\infty}$ and $F_{\infty}$ is important. A local variation in $\omega$ does not change the fiber of $F_{\infty}$ to which $\omega$ belongs but does change the corresponding fiber in $G_{\infty}$ since the resulting change in the "total" value of the extensive quantities is registered by $G_{\infty}$. Thus, for a given $\Delta X$ the change in the entropy, $\Delta S$, will depend on the value of $X$ at which the change is made. However, if it happens that $G_{\infty}$ is trivial then $\Delta S$ for a fixed $\Delta X$ has the same value almost everywhere and the state is a Gibbs state. In order to apply this kind of argument one has to be sure that it is possible to vary $X$ by an amount $\Delta X$ without being forced into an atypical configuration where $\Delta S$ would not have the above value and may not even be defined. This should not happen for a large enough collection of values of $\Delta \boldsymbol{X}$. For this, some "regularity" is crucial.

We shall therefore proceed by first decomposing a conditional equilibrium state to conditional equilibrium states with trivial $G_{\infty}$. If these retain the "regularity" properties, they are Gibbs states.

\section{a) The Decomposition}

The conditional probabilities given $G_{\infty}, \mu\left(\cdot \mid G_{\infty}\right)(\omega)$, provide us with the desired decomposition.

$$
\mu(\cdot)=\int \mu(d \omega) \mu\left(\cdot \mid G_{\infty}\right)(\omega)
$$

There are various properties of the decomposition which we shall need later. These can be proven by means of arguments given in Follmer [16] and Dynkin [17]. We shall therefore only state them and give a brief indication of their plausibility. It is convenient and justifiable to regard $\sigma$-algebras such as $G_{\infty}$ as partitions of $\Omega$ into fibers and the conditional measures like $\mu\left(d \bar{\omega} \mid G_{\infty}\right)(\omega)$ as the conditional probabilities given that $\bar{\omega}$ is in the fiber containing $\omega$.

P.1. $\mu\left(\cdot \mid G_{\infty}\right)(\omega)$ are C.E. states for $\mu$-a.e. $\omega$.

Since $G_{A} \supset G_{\infty}$, the expectation conditioned on $G_{A}$ in the state $\mu\left(\cdot \mid G_{\infty}\right)(\omega)$ is given by $\mu\left(\cdot \mid G_{A}\right)(\omega)$, i.e., by the corresponding microcanonical ensemble.

P.2. If $\mu$ is an extremal C.E. state then $G_{\infty}$ is trivial for $\mu$.

This follows from P.1 by means of the decomposition (4.1).

P.3. For $\mu$-a.e. $\omega G_{\infty}$ is $\mu\left(\cdot \mid G_{\infty}\right)(\omega)$-trivial.

P.4. If $G_{\infty}$ is trivial for a C.E. state $\mu$ then $\mu$ is a limit of the microcanonical ensembles $M_{A}(\cdot \mid \omega)$ for $\mu$-a.e. $\omega$. 
This follows from the convergence $G_{A} \uparrow G_{\infty}$, by means of Doob's martingale convergence theorem.

P.5. If $G_{\infty}$ is trivial for a C.E. state $\mu$ then $\mu$ is an extremal state.

Suppose $\mu=\alpha \mu_{1}+(1-\alpha) \mu_{2}$, then $G_{\infty}$ is trivial also for $\mu_{1} \cdot \mu$ and $\mu_{1}$ are therefore limits of the same sequence of microcanonical ensembles. Hence $\mu=\mu_{1}=\mu_{2}$.

Thus we have:

Theorem 4.1. A state is an extremal state of conditional equilibrium if and only if $G_{\infty}$ is trivial. Any state of conditional equilibrium may be decomposed into extremal C.E. states.

A word of caution is due here. If one decomposes a "regular" state of conditional equilibrium the resulting elements, while having the same expectations when conditioned on $G_{A}$, may no longer be "regular". In fact, this seemingly formal question relates to an essential property of infinite systems. It distinguishes them from finite systems for which the analogous statements are not true.

Example 4.1. Consider a system for which the total value of one of the extensive quantities such as $N(\omega)$ if finite for $\mu$-a.e. $\omega$. E.g., a system in a Gibbs state with an external potential, $V(q)$, which increases fast enough as $|q| \rightarrow \infty$. For such states $N(\omega)$ is constant on each element of the decomposition (4.1) into extremal C.E. states. After the decomposition, $\mu_{\Lambda}(\cdot \mid \omega)$ [i.e., $\left.\mu\left(\cdot \mid F_{\Lambda} G_{\infty}\right)(\omega)\right]$ are concentrated on $\left\{N_{A}=N_{A}(\omega)\right\}$ sets and one cannot vary $N_{A}$ without being forced into an atypical configuration.

\section{b) Extremal C.E. States and Gibbs States}

In order to state and prove our results we need to define some further regularity properties. The necessity for this is indicated by example 4.1 and by

Example 4.2. Consider a system of two species of particles (with occupation numbers $N_{1}$ and $N_{2}$ ). Let $e_{1}, e_{2} \in \mathbb{R}$ be incommensurable (i.e., $e_{1} / e_{2}$ is not rational) and let

$$
E=e_{1} N_{1}+e_{2} N_{2}
$$

Since in this case $N_{1, \Lambda}$ and $N_{2, \Lambda}$ can be determined from $E_{A}$, any state with

$$
S_{\Lambda}(\omega)=\lambda_{1} N_{1, \Lambda}(\omega)+\lambda_{2} N_{2, \Lambda}(\omega)+K_{\Lambda}
$$

is also a C.E. state for the single extensive quantity $E$. However, while $S_{\Lambda}-K_{\Lambda}$ is a function of $E_{A}$ it is not linear if $\lambda_{1} / e_{1} \neq \lambda_{2} / e_{2}$, and the state is not a Gibbs state for $E$ nor a combination of Gibbs states.

In this example, the distribution of $E$ is supported by an abelian group $\left(e_{1} \mathbb{Z}+e_{2} \mathbb{Z}\right)$ on which (measurable) additive functions need not be linear. In what follows, we impose conditions which require that the a priori distribution of our extensive quantities be compatible (in a sense made precise by the conditions) with a group on which additive functions are linear. This compatibility is expressed through conditions of absolute continuity with respect to the Haar measure of the group. The simplest such subgroups of $\mathbb{R}^{m}$ are of the form $\mathbb{R}^{k} \times \mathbb{Z}^{l}$. 
Notation. 1) In our considerations some of the extensive quantities have values in $\mathbb{R}$ (e.g., the energy $H$ ) and some in $\mathbb{Z}$ (e.g., the particle number $N$ ). We represent the collection of the extensive variables by $\boldsymbol{X}=(\boldsymbol{H}, \boldsymbol{N})$, their values by $\boldsymbol{u}=(\boldsymbol{e}, \boldsymbol{n})$ and their range space by $U=\mathbb{R}^{k} \times \mathbb{Z}^{l}(k+l=m)$.

2) The space on which $\hat{S}_{A}$ is naturally defined is $U \times \Omega$, equipped with the $\sigma$-algebra

$\hat{F}_{A}=$ Borel $\sigma$-algebra on $U \times F_{A}$.

The elements of $U$ act additively on $U \times \Omega$ by

$\boldsymbol{u}_{0}+(\boldsymbol{u}, \omega)=\left(\boldsymbol{u}+\boldsymbol{u}_{0}, \omega\right) \quad \forall \boldsymbol{u}_{0}, \boldsymbol{u} \in U, \omega \in \Omega$.

3) We shall use the maps:

$$
\begin{aligned}
g_{A}:(\Omega, \mathscr{B}) & \rightarrow\left(U \times \Omega, \hat{F}_{A}\right) \\
\omega & \mapsto\left(\boldsymbol{X}_{A}(\omega), \omega\right)
\end{aligned}
$$

and for $\bar{\Lambda}>\Lambda$, the maps

$$
\begin{aligned}
g_{\bar{\Lambda}, \Lambda}:\left(U \times \Omega, \hat{F}_{A}\right) & \rightarrow\left(U \times \Omega, \hat{F}_{\Lambda}\right) \\
g_{\bar{\Lambda}, \Lambda}:(\boldsymbol{u}, \omega) & \mapsto\left(\boldsymbol{u}+\boldsymbol{X}_{\bar{A}}\left(\omega_{\Lambda^{c}}\right), \omega\right) .
\end{aligned}
$$

These obey

$g_{\bar{A}}=g_{\bar{A}, A} g_{A}$.

4) We denote by $\tau_{A}$ the measure on $(\Omega, \mathscr{B})$ :

$$
\tau_{\Lambda}(d \omega)=v_{\Lambda}\left(d \omega_{A}\right) \mu\left(d \omega_{\Lambda^{c}}\right) .
$$

The map $g_{A}$ induces the measures $g_{A}(\mu)$ and $g_{A}\left(\tau_{A}\right)$ on $\left(U \times \Omega, \hat{F}_{A}\right)$.

A special role is played by the measure $m_{A}=$ (Lebesgue $\times$ counting) $\times \mu$ on $\hat{F}_{A}$.

5) The supports of the absolutely continuous parts of the measures $g_{A}(\mu)$ and $g_{\Lambda}\left(\tau_{A}\right)$ are defined by

$$
\begin{aligned}
& D_{A}=\sup \left\{B C \hat{F}_{A} \mid g_{A}(\mu) \approx m_{A} \quad \text { on } \quad \hat{F}_{A} \cap B\right\} \\
& =\left[\sup \left\{B \subset \hat{F}_{A} \mid g_{A}(\mu) \perp m_{A} \text { on } \quad \hat{F}_{A} \cap B\right\}\right]^{c} \\
& E_{A}=\sup \left\{B \subset \hat{F}_{A} \mid g_{A}\left(\tau_{A}\right) \approx m_{A} \text { on } \hat{F}_{A} \cap B\right\} \text {. }
\end{aligned}
$$

For $D_{A}$ "sup" should be understood in the sense of equivalence classes mod. $\left(m_{A}+g_{A}\left(\tau_{A}\right)\right) . \approx$ denotes the equivalence of measures and $\perp$ their mutual singularity.

The reason for introducing the above notation is that the entropy function $\hat{S}_{A}(\boldsymbol{u}, \omega)$, which is our main concern, is naturally defined on $U \times \Omega$ and is defined by $\mu$ only up to the measure $g_{A}\left(\tau_{A}\right)$. I.e., it is not defined for $(\boldsymbol{u}, \omega)$ which are not in the $\tau_{A}$-essential range of $g_{A}$. An example of this may be found in systems in which the interaction energy is positive. In such a case $\hat{S}$ is not defined for negative energies.

We shall consider the changes in entropy caused by variations in energy and particle number. In so doing one has to be assured that the new values are within the domain of definition of $\hat{S}$. At this point the continuity of translations with respect to Lebesgue measure becomes useful and the sets $D_{A}$ and $E_{A}$ enter. 


\section{Lemma 4.1.}

1) $g_{A}^{-1}\left(D_{A}\right), g_{A}^{-1}\left(E_{A}\right) \in G_{A}$.

2) $g_{A}^{-1}\left(D_{A}\right) \uparrow \bmod \mu$ and $g_{\Lambda}^{-1}\left(E_{A}\right) \uparrow \bmod \tau_{A}$ as $A \uparrow$.

3) $\bigcup_{\Lambda} g_{\Lambda}^{-1}\left(D_{\Lambda}\right), \bigcup_{\Lambda} g_{\Lambda}^{-1}\left(E_{\Lambda}\right) \in G_{\infty} \bmod \mu$.

Proof. We prove the above statements for the sets $E_{A}$. Replacing $\tau_{A}$ by $\mu$ one obtains a proof for the sets $D_{A}$.

1) $g_{A}:\left(\Omega, G_{A}\right) \rightarrow\left(U \times \Omega, \hat{F}_{\Lambda}\right)$ and $E_{\Lambda} \in \hat{F}_{\Lambda}$.

2) Let $\bar{\Lambda} \supset \Lambda$.

$$
g_{\bar{A}}^{-1}\left(E_{\bar{A}}\right)=g_{\bar{A}}^{-1} g_{\bar{A}, \Lambda}^{-1}\left(E_{\bar{A}}\right) \text {. }
$$

Thus it is enough to show

$$
g_{\bar{\Lambda}, \Lambda}^{-1}\left(E_{\bar{A}}\right) \supset E_{\Lambda} \bmod g_{\Lambda}\left(\tau_{A}\right) .
$$

By the definition of $E_{A}$ :

$$
g_{\bar{A}}\left(\tau_{\bar{A}}\right) \perp m_{\bar{A}} \text { on } \hat{F}_{\bar{A}} \cap E_{\bar{A}}^{c} \text {. }
$$

However $m_{\bar{A}}=m_{\Lambda}$ restricted to $\hat{F}_{\bar{A}}$ and $g_{\bar{\Lambda}, \Lambda}^{-1}\left(m_{\bar{A}}\right)=m_{\Lambda}$ on $g_{\bar{\Lambda},{ }_{A}}^{1}\left(\hat{F}_{\bar{\lambda}}\right) \subset \hat{F}_{\Lambda}$. By the regularity of $\mu: \tau_{A} \ll \tau_{\bar{A}}$, hence

$$
g_{\Lambda}\left(\tau_{\Lambda}\right)=g_{\bar{A}, A}^{-1} g_{\bar{A}}\left(\tau_{\Lambda}\right) \ll g_{\bar{A}, \Lambda}^{-1} g_{\bar{A}}\left(\tau_{\tilde{A}}\right) \quad \text { on } \quad g_{\bar{A}, \Lambda}^{1}\left(\hat{F}_{\bar{A}}\right) .
$$

Therefore

$$
g_{A}\left(\tau_{A}\right) \perp m_{\Lambda} \quad \text { on } \quad g_{\bar{A}, \Lambda}^{-1}\left(\hat{F}_{\bar{A}}\right) \cap g_{\overline{\bar{A}}, \Lambda}^{-1}\left(E_{\bar{A}}^{c}\right),
$$

and thus also on $\hat{F}_{\Lambda} \cap g_{\bar{\Lambda}}^{-1}{ }_{\Lambda}^{1}\left(E_{\bar{A}}^{c}\right)$. When compared with the definition of $E_{\Lambda}$ this implies $g_{\overline{\bar{A}}, \Lambda}^{-1}\left(E_{\bar{\Lambda}}\right)^{c}=g_{\bar{\Lambda}, \Lambda}^{-1}\left(E_{\bar{A}}^{c}\right) \subset E_{\Lambda}^{c}$, i.e., $g_{\bar{A},{ }_{A}}^{-1}\left(E_{\bar{A}}\right) \supset E_{\Lambda} \bmod g_{\Lambda}\left(\tau_{A}\right)$.

3) $\forall \bar{\Lambda}:$

$$
\bigcup_{\Lambda} g_{\bar{A}}^{-1}\left(E_{\Lambda}\right)=\bigcup_{\bar{A} \supset \bar{A}} g_{\overline{\bar{A}}}^{-1}\left(E_{\bar{\Lambda}}\right) \in G_{\bar{A}} \bmod \mu
$$

thus

$$
\bigcup_{\Lambda} g_{\bar{A}}^{-1}\left(E_{\Lambda}\right) \in \bigcap_{\bar{A}} G_{\bar{A}}=G_{\infty} \bmod \mu .
$$

For extremal C.E. states $G_{\infty}$ is trivial. In such a state $\mu$ either $\mu\left(g_{\Lambda}^{-1}\left(D_{\Lambda}\right)\right) \underset{\Lambda \uparrow \infty}{\longrightarrow} 1$ or $\mu\left(G_{\Lambda}^{-1}\left(D_{\Lambda}\right)\right)=0 \forall A$.

We now formulate a regularity condition which is violated in the latter case. It assures the possibility of "adding a particle" to a typical (and non-singular) configuration (and therefore, if $G_{\infty}$ is trivial, to almost all configurations), without obtaining an atypical configuration.

Definition 4.1. A state $\mu$ is loose if $\exists \Lambda$ such that, for some $e_{1}, \ldots, e_{l} \in \mathbb{R}^{k}$

$$
\mu\left(g_{\Lambda}^{-1}\left(D_{A} \cap\left(\left(\boldsymbol{e}_{i}, \mathbf{1}_{i}\right)+D_{A}\right)\right)\right) \neq 0
$$

with $\mathbf{1}_{1}=(1,0,0, \ldots), \mathbf{1}_{2}=(0,1,0, \ldots) \ldots$.

Note that since $D_{A}$ is the support of the absolutely continuous part of $g_{A}(\mu)$, $\mu\left(g_{\Lambda}^{-1}\left(D_{\Lambda}\right)\right) \neq 0$ assures that the energy can be varied continuously.

The main results of this section are the following two theorems. 
Theorem 4.2. Let $\mu$ be a loose regular extremal C.E. state. Then there exists $\lambda \in \mathbb{R}^{m}$ such that for any $\Lambda$

$$
S_{\Lambda}(\omega)= \begin{cases}\lambda \cdot \boldsymbol{X}_{\Lambda}(\omega)+K_{\Lambda}\left(\omega_{\Lambda^{c}}\right) & \omega \in \bigcup_{\Lambda \subset \bar{\Lambda} \uparrow \mathbb{R}^{d}} g_{\bar{\Lambda}}^{-1}\left(E_{\bar{\Lambda}}\right) \\ \infty & \text { otherwise }\end{cases}
$$

for $\tau_{A}$-a.e. $\omega$, where the $K_{\Lambda}$ are some $F_{A}$ measurable functions.

Theorem 4.3. Let $\mu$ be a loose regular extremal C.E. state with respect to $\{\boldsymbol{H}, \boldsymbol{N}\}, N_{i}$ $\geqq 0 \forall i$. If, furthermore, for all sufficiently large $\Lambda$ the distribution of $H\left(\xi \mid \omega_{\Lambda^{c}}\right)$ obtained from $v_{A}(d \xi)$ on $\left\{N_{i, \Lambda}(\xi)>0, \forall i\right\}$ is absolutely continuous with respect to Lebesgue measure for $\mu$-a.e. $\omega$ (i.e., $g_{A}\left(\tau_{A}\right) \ll m_{A}$ on $\left\{\boldsymbol{N}_{A}>0\right\}$ ) then $\mu$ is a Gibbs state.

Definition 4.2. Let $\boldsymbol{x} \in U$.

1) $Q_{\Lambda}^{\boldsymbol{x}}=g_{\Lambda}^{-1}\left(D_{\Lambda} \cap\left(-\boldsymbol{x}+E_{\Lambda}\right)\right) \quad\left(\in G_{\Lambda}\right)$.

2) For $\omega \in Q_{\Lambda}^{x}$,

$d_{\Lambda}(\boldsymbol{x}, \omega)=\hat{S}_{\Lambda}\left(\boldsymbol{x}+g_{\Lambda}(\omega)\right)-\hat{S}_{\Lambda}\left(g_{\Lambda}(\omega)\right)$.

On its domain, $d_{\Lambda}(\boldsymbol{x}, \cdot)$ is defined $\bmod \tau_{\Lambda}$ and takes values in $(-\infty, \infty]$.

\section{Lemma 4.2.}

1) $Q_{\Lambda}^{x} \uparrow \bmod \mu$ as $\Lambda \uparrow$.

2) $\operatorname{On} Q_{\Lambda}^{\boldsymbol{x}}, d_{\bar{\Lambda}}(x, \omega)=d_{\Lambda}(\boldsymbol{x}, \omega) \bmod \mu$.

Proof. 1) $g_{\bar{\Lambda} . \Lambda}$ commutes with the addition of $\boldsymbol{x}$. Therefore

$$
Q_{\Lambda}^{\boldsymbol{x}}=g_{\bar{\Lambda}}^{-1}\left(D_{\Lambda}\right) \cap g_{\Lambda}^{-1}\left(-\boldsymbol{x}+g_{\overline{\bar{A}}, \Lambda}^{-1}\left(E_{\bar{\Lambda}}\right)\right) .
$$

Since by Lemma 4.1 and its proof $g_{\bar{\Lambda}, \Lambda}^{-1}\left(E_{\bar{A}}\right) \supset E_{A}$ and $g_{\bar{\Lambda}}^{-1}\left(D_{\bar{\Lambda}}\right) \supset g_{\bar{A}}^{-1}\left(D_{A}\right), Q_{\bar{\Lambda}}^{\boldsymbol{x}} \supset Q_{\Lambda}^{\boldsymbol{x}}$.

2) By Lemma 3.1, for $m_{\Lambda}$-a.e. $(\boldsymbol{y}, \omega) \in D_{\Lambda} \cap\left(-\boldsymbol{x}+E_{\Lambda}\right)$,

$$
\hat{S}_{\bar{\Lambda}}\left(g_{\bar{\Lambda}, \Lambda}(\boldsymbol{y}, \omega)\right)=\hat{S}_{\Lambda}(\boldsymbol{y}, \omega)+C_{\bar{\Lambda}, \Lambda}\left(\omega_{\Lambda^{c}}\right)
$$

and

$$
\hat{S}_{\bar{\Lambda}}\left(g_{\bar{\Lambda}, \Lambda}(\boldsymbol{x}+\boldsymbol{y}, \omega)\right)=\hat{S}_{\Lambda}(\boldsymbol{x}+\boldsymbol{y}, \omega)+C_{\bar{\Lambda}, \Lambda}\left(\omega_{\Lambda^{c}}\right) .
$$

Furthermore, all the terms in the first equation are finite so that we may subtract it from the second equation.

Lemma 4.3. Under the assumptions of Theorem 3, $\forall \boldsymbol{x} \in U$

$$
\mu\left(Q_{\Lambda}^{\boldsymbol{x}}\right) \rightarrow 1 \quad \text { as } \quad \Lambda \uparrow \mathbb{R}^{d}
$$

Proof. Let

$$
J_{A}=\left\{\boldsymbol{x} \in U \mid \mu\left(Q_{A}^{\boldsymbol{x}}\right)>0\right\} .
$$

Since $g_{\Lambda}(\mu) \approx m_{\Lambda}$ on $D_{\Lambda}$

$J_{A}=\left\{\boldsymbol{x} \in U \mid m_{\Lambda}\left(D_{A} \cap\left(-\boldsymbol{x}+E_{A}\right)\right)>0\right\}$.

$J_{A}$ is open since $m_{\Lambda}\left(D_{\Lambda} \cap\left(-\boldsymbol{x}+E_{\Lambda}\right)\right)$ is continuous in $\boldsymbol{x}$ (a well known property of Lebesgue measure). Since $\mu$ is loose, for any $i=1, \ldots, l, J_{\Lambda} \ni\left(\boldsymbol{e}_{i},-\mathbf{1}_{i}\right)$ eventually for some $\Lambda$ and $\boldsymbol{e}_{i} \in \mathbb{R}^{k}$. 
By Lemma 4.2 the $J_{\Lambda}$ increase in $\Lambda$ to some set $J$ (i.e., $J_{A} \uparrow J$ ). $\forall \boldsymbol{x} \in J$,

$\bigcup_{\Lambda} Q_{\Lambda}^{\boldsymbol{x}}=\lim _{\Lambda \uparrow \mathbb{R}^{d}} Q_{\Lambda}^{\boldsymbol{x}} \in G_{\infty} \bmod \mu$.

Since $G_{\infty}$ is trivial, the above is a trivial set of positive measure. Thus $\forall \boldsymbol{x} \in J$,

$\lim _{\Lambda \uparrow \mathbb{R}^{d}} \mu\left(Q_{\Lambda}^{\boldsymbol{x}}\right)=1$.

Let now $\boldsymbol{x}, \boldsymbol{x}+\boldsymbol{y} \in J . \exists \Lambda$ for which

$\mu\left(Q_{\Lambda}^{\boldsymbol{x}+y} \cap Q_{\Lambda}^{\boldsymbol{x}}\right) \neq 0$

or

$\left(g_{\Lambda} \mu\right)\left(D_{\Lambda} \cap\left(-\boldsymbol{x}+D_{\Lambda}\right) \cap\left(-\boldsymbol{x}-\boldsymbol{y}+E_{A}\right)\right)>0$.

As explained above this means

$m_{\Lambda}\left(D_{\Lambda} \cap\left(-\boldsymbol{x}+D_{\Lambda}\right) \cap\left(-\boldsymbol{x}-\boldsymbol{y}+E_{\Lambda}\right)\right)>0$,

which implies, using the translational invariance of $m_{\Lambda}$,

$m_{A}\left(D_{A} \cap\left(-\boldsymbol{y}+E_{A}\right)\right)>0$

i.e., $\boldsymbol{y} \in J$.

Therefore, $J$ is an open subset of $\mathbb{R}^{k} \times \mathbb{Z}^{l}$ which is closed under subtraction and which contains points $\left(\boldsymbol{e}_{i},-\mathbf{1}_{i}\right) i=1, \ldots, l$. It follows that $J=\mathbb{R}^{k} \times \mathbb{Z}^{l}$, which proves the lemma.

Lemma 4.4. Under assumptions of Theorem $3 \exists \lambda \in \mathbb{R}^{m}$ such that $\forall \boldsymbol{x} \in U \forall \Lambda$ and $\mu$-a.e. $\omega \in Q_{\Lambda}^{x}$

$$
d_{\Lambda}(\boldsymbol{x}, \omega)=\lambda \cdot \boldsymbol{x} .
$$

Proof. By Lemma 4.2, $\forall \boldsymbol{x} \in U$ there exists the limiting function $d$ defined by

$$
\lim _{\Lambda \uparrow \mathbb{R}^{d}} d_{\Lambda}(\boldsymbol{x}, \omega)=d(\boldsymbol{x}, \omega) .
$$

$d(\boldsymbol{x}, \omega)$ is measurable $\bmod \mu$ with respect to $G_{\infty}$ and thus, $\bmod \mu$, is a constant $d(\boldsymbol{x})$.

This implies, since $g_{A}(\mu) \approx m_{A}$ on $D_{A}$, that for $m_{A}$ a.e. $(\boldsymbol{u}, \omega) \in D_{A} \cap\left(-\boldsymbol{x}+E_{A}\right)$

$\hat{S}_{\Lambda}(\boldsymbol{x}+\boldsymbol{u}, \omega)-\hat{S}_{\Lambda}(\boldsymbol{u}, \omega)=d(\boldsymbol{x})$.

Let $\boldsymbol{x}, \boldsymbol{y} \in U$. In the proof of Lemma 4.3 it was shown that $\exists \Lambda$ such that

$$
m_{\Lambda}\left(D_{\Lambda} \cap\left(-\boldsymbol{x}+D_{\Lambda}\right) \cap\left(-\boldsymbol{x}-\boldsymbol{y}+E_{\Lambda}\right)\right) \neq 0 .
$$

Using the translation of $m_{A}, \quad(4.2)$ implies, for $m_{A}$-a.e. $(\boldsymbol{u}, \omega) \in D_{A}$ $\cap\left(-\boldsymbol{x}+D_{A}\right) \cap\left(-\boldsymbol{x}-\boldsymbol{y}+E_{\Lambda}\right)$

$$
\begin{aligned}
\hat{S}(\boldsymbol{x}+\boldsymbol{u}, \omega)-\hat{S}(\boldsymbol{u}, \omega) & =d(\boldsymbol{x}), \\
\hat{S}(\boldsymbol{x}+\boldsymbol{y}+\boldsymbol{u}, \omega)-\hat{S}(\boldsymbol{u}, \omega) & =d(\boldsymbol{x}+\boldsymbol{y}), \\
\hat{S}(\boldsymbol{x}+\boldsymbol{y}+\boldsymbol{u}, \omega)-\hat{S}(\boldsymbol{x}+\boldsymbol{u}, \omega) & =d(\boldsymbol{y}) .
\end{aligned}
$$

Hence $d(\boldsymbol{x})+d(\boldsymbol{y})=d(\boldsymbol{x}+\boldsymbol{y}) \forall \boldsymbol{x}, \boldsymbol{y} \in U$. The joint measurability of $d(\boldsymbol{x}, \omega)$ implies that $d(\boldsymbol{x})=\int \mu(d \omega) d(\boldsymbol{x}, \omega)$ is measurable. Therefore $d$ is linear. 
Proof of Theorem 4.2. Consider configurations $\omega$ for which

$$
\mu\left(g_{\Lambda}^{-1}\left(D_{\Lambda}\right) \mid F_{\Lambda}\right)(\omega)>0 .
$$

By Lemma 4.4 (and Fubini's theorem), $\exists \lambda \in \mathbb{R}^{m}$ such that $\forall \Lambda$ the $\hat{F}_{\Lambda}$-measurable function

$$
\hat{S}_{\Lambda}(\boldsymbol{x}, \omega)-\boldsymbol{\lambda} \cdot \boldsymbol{x}
$$

is independent of $\boldsymbol{x}$ for $m_{\Lambda}$-a.e. $(\boldsymbol{x}, \omega) \in E_{\Lambda}$. This difference is therefore given by some function, $K_{A}\left(\omega_{A^{c}}\right)$.

It follows from Lemma 3.1 that $\forall \Lambda \subset \bar{\Lambda}$

$$
\begin{aligned}
\hat{S}_{\Lambda}(\boldsymbol{x}, \omega)-\boldsymbol{\lambda} \cdot \boldsymbol{x} & =\hat{S}_{\bar{\Lambda}}\left(g_{\bar{\Lambda}, \Lambda}(\boldsymbol{x}, \omega)\right)-\boldsymbol{\lambda} \cdot \boldsymbol{x}-C_{\bar{\Lambda}, \Lambda}\left(\omega_{\Lambda^{c}}\right) \\
& =\hat{S}_{\bar{\Lambda}}\left(\boldsymbol{x}+\boldsymbol{X}_{\bar{\Lambda}}\left(\omega_{\Lambda^{c}}\right), \omega\right)-\boldsymbol{\lambda} \cdot \boldsymbol{x}-C_{\bar{\Lambda}_{\Lambda}}\left(\omega_{\Lambda^{c}}\right) \\
& =K_{\bar{\Lambda}}\left(\omega_{\bar{\Lambda}^{c}}\right)+\boldsymbol{\lambda} \cdot \boldsymbol{X}_{\bar{\Lambda}}\left(\omega_{\Lambda^{c}}\right)-C_{\bar{\Lambda}, \Lambda}\left(\omega_{\Lambda^{c}}\right)
\end{aligned}
$$

for $g_{A}\left(\tau_{A}\right)$-a.e. $\quad(\boldsymbol{x}, \omega) \in\left\{\omega \mid \mu\left(g_{\bar{A}}^{-1}\left(D_{\bar{A}}\right) \mid F_{\bar{A}}\right)(\omega)>0\right\} \cap g_{\bar{A}, \Lambda}^{-1}\left(E_{\bar{A}}\right)$. [Since $g_{\bar{A},{ }_{A}} g_{A}\left(\tau_{A}\right)$ $=g_{\bar{\Lambda}}\left(\tau_{\Lambda}\right) \ll g_{\bar{A}}\left(\tau_{\bar{A}}\right)$.] This proves that $S_{\Lambda}(\omega)$ has the claimed form on the union of increasing sets :

$$
\bigcup_{\Lambda \subset \tilde{A}}\left[\left\{\omega \mid \mu\left(g_{\bar{A}}^{-1}\left(D_{\bar{A}}\right) \mid F_{\bar{A}}\right)(\omega)>0\right\} \cap g_{\overline{\bar{A}}}^{-1}\left(E_{\bar{\Lambda}}\right)\right] .
$$

Since

$$
\mu\left(\left\{\omega \mid \mu\left(g_{\overline{\bar{A}}}^{-1}\left(D_{\bar{A}}\right) \mid F_{\bar{A}}\right)(\omega)>0\right\}\right)>1-\mu\left(g_{\bar{A}}^{-1}\left(D_{\bar{A}}\right)\right)
$$

and $\mu\left(D_{\bar{\Lambda}}\right) \rightarrow 1$ as $\bar{\Lambda} \uparrow \mathbb{R}^{d}$,

$$
\mu\left(\bigcup_{\Lambda \subset \bar{A}}\left[\left\{\omega \mid \mu\left(g_{\bar{A}}^{-1}\left(D_{\bar{A}}\right) \mid F_{\bar{A}}\right)(\omega)>0\right\}\right]\right)=1 .
$$

Further, since $\mu\left(g_{\bar{A}}^{-1}\left(D_{\bar{A}}\right) \mid F_{\bar{A}}\right)(\omega)$ is measurable with respect to $F_{\bar{A}} \subset F_{A}$, the above set has the "full measure" with respect to $\tau_{\Lambda}$. The above conclusion extends therefore to the set $\bigcup_{\mathcal{A} \subset \bar{A}} g_{\bar{A}}^{-1}\left(E_{\bar{A}}\right)$. It remains only to prove that on its complement $S_{\Lambda}(\omega)=\infty\left(\bmod \tau_{\Lambda}\right)$. This follows from the fact that

$$
\mu\left(\bigcup_{\Lambda}\left(g_{\Lambda}^{-1}\left(E_{\Lambda}\right)\right)^{c}\right)=0
$$

Proof of Theorem 4.3. By Theorem 4.2 it suffices to show that

$$
\tau_{A}\left(\left[\bigcup_{\Lambda \subset \bar{A}} g_{\bar{A}}^{-1}\left(E_{\bar{A}}\right)^{c}\right)=0 .\right.
$$

Since $g_{\bar{A}}\left(\tau_{\bar{A}}\right) \ll m_{\bar{A}}$ on $\left[\bigcup_{i=1}^{l}\left\{N_{i, \bar{A}}=0\right\}\right]^{c}$, for any $\bar{\Lambda}$ large enough,

$$
\left[\bigcup_{\Lambda \subset \bar{A}} g_{\overline{\bar{A}}}^{-1}\left(E_{\bar{A}}\right)\right]^{c} \subset \bigcap_{\Lambda \subset \bar{A}}\left(\bigcup_{i=1}^{l}\left\{N_{i, \bar{\Lambda}}=0\right\}\right)=\bigcup_{i=1}^{l}\left\{N_{i}=0\right\} \bmod \tau_{\Lambda} .
$$

Here $N_{i}=\lim _{\bar{A} \rightarrow \mathbb{R}^{d}} N_{i, \bar{A}}$.

Suppose now $\mu\left\{N_{i}-N_{i, A}=0\right\}>0$ for some $1 \leqq i \leqq l$. Then, for some $n<\infty$,

$$
\mu\left\{N_{i}=n\right\}>0 \text {. }
$$


However, $\left\{N_{i}=n\right\} \in G_{\infty}$ and $G_{\infty}$ is trivial by the extremality of $\mu$. This implies

$$
N_{i}=n \bmod \mu \text {, }
$$

which contradicts the looseness of $\mu$. Thus $\mu\left\{N_{i}-N_{i, \Lambda}=0\right\}=0$ and hence $\tau_{\Lambda}\left(\bigcup_{i=1}^{l}\left\{N_{i}-N_{i, \Lambda}=0\right\}\right)=0$

\section{Application to Statistical Mechanical Systems}

We shall now restrict our attention to systems similar to those described in Remark 2.1. Namely, the extensive variables are $X=\{H, N\}$ where the Hamiltonian $H$ consists of

1) The kinetic energy $\Sigma_{p^{2} / 2 m}$.

1) Potential energy of the form $V=\sum_{k=1}^{n} \Sigma_{f_{k}}$

with $k$-body potentials $f_{k}$ which are upper semi-continuous of compact support with values in $\mathbb{R} \cup\{+\infty\}$ (one side compactification of $\mathbb{R}$ ).

3) Hard core exclusion is also permitted.

Definition 5.1. 1) For a given Hamiltonian let $\phi(q \mid \omega)$ denote the increase in the interaction energy which would result from adding a particle at $q \in \mathbb{R}^{d}$ to the configuration $\omega \in \Omega$.

2) A state $\mu$ is called unsaturated if for $\mu$-a.e. $\omega \exists \gamma \in \mathbb{R}$ such that

$$
g_{\gamma}(\omega)=\lim _{\Lambda \rightarrow \mathbb{R}^{d}} \inf N_{\Lambda}(\omega)^{-1} \int_{\Lambda} d q I[\phi(q \mid \omega)<\gamma]>0 .
$$

Remark 5.1. It is easy to prove the following quite general criteria for unsaturated states.

1) Let the potential energy result from a pair interaction $f_{2}$ such that $\forall \varepsilon>0$

$$
A(\varepsilon)=\sup _{x} \int_{|y-x|>\varepsilon} d y\left|f_{2}(x, y)\right|<\infty \text {. }
$$

If for $\mu$-a.e. $\omega$

$$
\boldsymbol{\varrho}=\lim _{\Lambda \rightarrow \mathbb{R}^{d}} \inf \frac{N_{\Lambda}(\omega)}{|\Lambda|}<\infty
$$

then $\mu$ is unsaturated.

Proof. Let $\omega_{A}=\left\{\left(q_{i}, p_{i}\right)\right\}$.

$$
\begin{aligned}
\int_{\Lambda} d q I[\phi(q \mid \omega)>\gamma] & \leqq N_{\Lambda}(\omega) \int_{|q| \leqq \varepsilon} d q+\int_{\forall i:\left|q-q_{2}\right|>\varepsilon} d q|\phi(q \mid \omega)| / \gamma \\
& \left.\leqq N_{\Lambda}(\omega) \int_{|q| \leqq \varepsilon} d q+\gamma^{-1} \sum_{i} \int_{\left|q-q_{i}\right|<\varepsilon} d q \mid f_{2}\left(q-q_{i}\right)\right\} \\
& \leqq N_{\Lambda}(\omega) \int_{|q| \leqq \varepsilon} d q+N_{\Lambda}(\omega) A(\varepsilon) / \gamma .
\end{aligned}
$$

Therefore

$$
\lim _{\Lambda \rightarrow \mathbb{R}^{d}} \sup N_{\Lambda}(\omega)^{-1} \int_{\Lambda} d q I[\phi(q \mid \omega)<\gamma] \geqq \varrho^{-1}-\frac{A(\varepsilon)}{\gamma}-\int_{|q| \leqq \varepsilon} d q
$$

which is positive for properly chosen $\varepsilon$ and $\gamma$. 
2) Let $H$ and $\mu$ be translation invariant with $\int d \mu N_{\Lambda}(\omega)<\propto . \mu$ is saturated (the opposite of unsaturated) if and only if, $\forall q \in \mathbb{R}^{d}, \phi(q, \omega)=\infty$ for $\mu$-a.e. $\omega$ (this can be proven using the decomposition to ergodic components).

In particular, if the potential energy results from a pair potential which is finite almost everywhere then $\mu$ is unsaturated.

Our main result in this section is

Theorem 5.1. Let $\mu$ be a conditional equilibrium state with respect to $\boldsymbol{X}=\{H, N\}$ with $H$ as above. If $\mu$ is unsaturated and for $\mu$-a.e. $\omega$

$$
\hat{p}^{2}(\omega)=\lim _{\Lambda \rightarrow \mathbb{R}^{d}} \inf \frac{\Sigma_{p^{2}}\left(\omega_{\Lambda}\right)}{N_{\Lambda}(\omega)}>0
$$

and

$$
N_{\mathbb{R}^{d}}(\omega)=\infty
$$

then $\mu$ is a convex combination of Gibbs states.

We shall prove this using the criterion provided by Theorem 4.3. First let us observe:

Lemma 5.1 (The Three-Boxes Criterion). Let $\mu$ be an extremal C.E. state with respect to $\boldsymbol{X}=\{H, N\}$ with $H$ as above. If for $\mu$-a.e. $\omega$ the measure on $\mathbb{R} \times \mathbb{Z}$ defined by the weak $\lim _{\overline{\bar{A}} \uparrow \mathbb{R}^{d}} \lim _{\overline{\bar{A} \uparrow \mathbb{R}^{d}}}$ of the distributions of $\left(H_{\Lambda}(\bar{\omega}), N_{\Lambda}(\bar{\omega})\right)$ in the states

$$
\mu\left(d \bar{\omega} \mid \mathscr{B}_{\bar{\Lambda} \backslash \Lambda} G_{\bar{\Lambda}}\right)(\omega)
$$

is

i) absolutely continuous on $\mathbb{R} \times\{n>0\}$ for all $\Lambda$, and

ii) for $\Lambda$ large enough, equivalent to (Lebesgue $\times$ counting) measure in a set of the form $\left(H_{\Lambda}(\omega)-\Delta, H_{\Lambda}(\omega)\right) \times\left\{N_{\Lambda}(\omega)-1, N_{\Lambda}(\omega)\right\}, \Delta>0$, then $\mu$ is a Gibbs state.

Proof. Let

$$
L_{\Lambda, \bar{A}}=\bigcap_{\bar{\Lambda}} \mathscr{B}_{\bar{\Lambda} \backslash \Lambda} G_{\bar{\Lambda}}
$$

and

$$
\bar{L}_{\Lambda}=\bigcup_{\Lambda} L_{A, \bar{X}} .
$$

It is a consequence of Doob's theorem that the limiting distribution on $\mathbb{R} \times \mathbb{Z}$ is the distribution of $\left(H_{A}, N_{A}\right)$ in the state $\mu(\cdot \mid \bar{L})(\omega)$.

$$
L_{\Lambda, \bar{\Lambda}} \supset \mathscr{B}_{\bar{\Lambda} \backslash \Lambda} G_{\infty}
$$

and, since the product of $\sigma$-algebras is continuous with respect to upward convergence,

$$
\bar{L}_{\Lambda} \supset \lim _{\bar{A} \uparrow \mathbb{R}^{d}}^{-} \mathscr{B}_{\bar{\Lambda} \backslash A} G_{\infty}=F_{\Lambda} G_{\infty}=F_{A},
$$

the last equality only $\bmod \mu$, (because $G_{\infty}$ is trivial by the extremality of $\mu$ ). This implies that the measures $\mu\left(\cdot \mid F_{A}\right)(\omega)$ are averages over $\mu\left(\cdot \mid \bar{L}_{A}\right)(\bar{\omega})$ and hence conditions i) and ii) are also satisfied by the distributions of $\left(H_{\Lambda}, N_{\Lambda}\right)$ in the states 
$\mu\left(\cdot \mid F_{A}\right)(\omega)$. Therefore, except possibly for regularity, $\mu$ satisfies the hypotheses of Theorem 4.3.

In order to prove regularity it suffices, since $\mu$ is a C.E. state, to show

$$
g_{\Lambda}(\mu) \ll g_{A}\left(\tau_{A}\right) \text {. }
$$

This is clearly satisfied on $\{n \leqq 0\}$. On $\{n>0\} \quad g_{\Lambda}(\mu) \ll m_{\Lambda}$ (by i) and $g_{\Lambda}(\mu)$ is supported by $\left\{e \geqq e_{\Lambda}^{0}(n, \omega)\right\}$ where

$$
e_{\Lambda}^{0}(n, \omega)=\inf \left\{H_{\Lambda}(\bar{\omega}) \mid N_{\Lambda}(\bar{\omega})=n, \bar{\omega}_{\Lambda^{c}}=\omega_{\Lambda^{c}}\right\}=\inf \left\{V\left(q_{1}, \ldots, q_{n} \mid \omega\right) .\right.
$$

Since the kinetic energy and the potential energy are independent with respect to $v_{A}$, and since the distribution of the kinetic energy is equivalent to the Lebesgue measure on $[0, \infty)$, we conclude that $g_{\Lambda}\left(\tau_{\Lambda}\right) \approx m_{\Lambda}$ on

$$
\left\{e \geqq \operatorname{ess} \inf \left\{V\left(q_{1}, \ldots, q_{m} \mid \omega_{\Lambda^{c}}\right) \mid q_{1}, \ldots, q_{n} \in \Lambda\right\}\right\} .
$$

By the upper semi-continuity of the interactions this essential infimum is $e_{\Lambda}^{0}(n, \omega)$. Thus on $\left\{e \geqq e_{\Lambda}^{0}(n, \omega)\right\}$

$$
g_{\Lambda}(\mu) \ll m_{\Lambda} \ll g_{\Lambda}\left(\tau_{\Lambda}\right) \text {. }
$$

The lemma now follows from Theorem 4.3.

We wish to point out that the extremality of $\mu$ does not play an essential role in the above proof; hence the other conditions of Lemma 5.1 provide a criterion for a C.E. state to be a convex integral of Gibbs states.

Proof of Theorem 5.1. Let $\mu$ satisfy the assumptions of the theorem. Then so do the components of its decomposition into extremal C.E. states. It thus suffices to prove the theorem for $\mu$ extremal.

For any $D \subset \mathbb{R}^{d}, e \in \mathbb{R}, n \in \mathbb{Z}$ such that $n d>2$ let

$$
\begin{aligned}
R_{D}(e, n \mid \omega) & =\frac{d}{d e} \int_{q \in D^{d}} d^{n d} q \int_{p^{2}<e-v\left(q \mid \omega_{D} c\right)} d^{n d} p \\
& =c_{d} \int_{\substack{q \in D^{d} \\
e-V\left(q \mid \omega_{D}\right)>0}} d^{n d} q \int_{|p|=\sqrt{e-V}} \sigma^{n d}(d p)|p|^{-1} .
\end{aligned}
$$

The interchange of integration and differentiation is justified since $V$, being upper semicontinuous, is bounded below on compact sets.

For given $\Lambda \subset \bar{\Lambda} \subset \overline{\bar{\Lambda}} \subset \mathbb{R}^{d}$ let

$$
D=\overline{\bar{\Lambda}} \backslash \bar{\Lambda} \quad \text { and } \quad \bar{D}=\Lambda \cup D \text {. }
$$

The dependence of $D$ on $\Lambda$ will be supressed in this notation.

If, as we shall assume, $\bar{\Lambda} \backslash \Lambda$ is wider than the range of the interaction then

$$
H_{\bar{D}}(\omega)=H_{\Lambda}(\omega)+H_{D}(\omega) \text {. }
$$

The probability of finding $\left(H_{\Lambda}(\bar{\omega}), N_{A}(\bar{\omega})\right)$ in $d e \times\{n\}$ with respect to

$$
\mu\left(d \bar{\omega} \mid \mathscr{B}_{\bar{\Lambda} \backslash \Lambda} G_{\bar{\Lambda}}\right)(\omega)
$$

is, for $n \in\left(2 / d, N_{D}(\omega)-2 / d\right)$,

$$
(\text { Norm })^{-1}(d e / n !) R_{\Lambda}(e, n \mid \omega) W_{D, \Lambda}(e, n \mid \omega)
$$

with

$$
W_{D, \Lambda}(e, n \mid \omega)=\left(N_{\bar{D}}(\omega)-n\right) !^{-1} R_{D}\left(H_{\bar{D}}(\omega)-e, N_{\bar{D}}(\omega)-n \mid \omega\right) .
$$


We aim at showing that the hypotheses of Lemma 5.1 are satisfied. In order to do so we need some estimates on the effects on the above distribution of changes in $e$ and $n$ which are uniform in (large) $\bar{\Lambda}$ and $\bar{\Lambda}$. These are provided by the following monotonicity properties of the weight function $W$.

Claim 1. i) $W_{D, A}(e, n \mid \omega)$ is decreasing in $e$.

ii) $\exists c>0$ such that for all $\bar{\Lambda} \supset \Lambda$ there is a sequence $\overline{\bar{\Lambda}} \uparrow \mathbb{R}^{d}$ for which

$\lim \inf \frac{W_{D, \Lambda}\left(H_{\Lambda}(\omega), N_{\Lambda}(\omega)-1 \mid \omega\right)}{W_{D, \Lambda}\left(H_{\Lambda}(\omega), N_{\Lambda}(\omega) \mid \omega\right)} \geqq c \bmod \mu$.

i) follows by inspection of Equation (5.4). ii) will now be proven.

Letting $E=H_{\bar{D}}(\omega)-e, M=N_{\bar{D}}(\omega)-n$ and $\phi(\bar{q} \mid q)=\phi\left(\bar{q} \mid q, \omega_{D^{c}}\right)$,

$$
\begin{aligned}
W_{D, \Lambda}(e, n-1 \mid \omega)= & \left.(M+1) !^{-1} \frac{d}{d e}\right|_{E} \int_{\bar{q} \in D} d^{d} \bar{q} \int d^{d} \bar{p} \\
& \cdot \int_{q \in D^{M}} d^{M d} q \int_{p^{2}<e-V(q \mid \omega)-\bar{p}^{2}-\phi(\bar{q})} d^{M d} p \\
= & c_{d}(M+1) !^{-1} \int_{E-V(q \mid \omega)-\bar{p}^{2}-\phi(\bar{q}) \geqq 0} d^{d} \bar{q} \int d^{d} \bar{p} \int d^{M d} q \\
& \int_{|p|=\sqrt{E-V-\bar{p}^{2}-\phi} \sigma^{M d}(d p)|p|^{-1}} \\
\geqq & c_{d}(M+1) !^{-1} \int_{E-V \geqq 0} d^{M d} q \int_{|p|=\sqrt{E-V}} \sigma^{M d}(d p)|p|^{-1} \\
& \cdot \quad \begin{array}{l}
d^{d} \bar{q} \int d^{d} \bar{p} \\
E-V-\bar{p}^{2}-\phi \geqq 0
\end{array}
\end{aligned}
$$

The last inequality results from the additional restriction of the domain of integration and from scaling $p$. The distribution of $q, p$ in the last expression corresponds to the microcanonical ensemble in $D,\langle\cdot\rangle_{\mu, E, \omega}$, for $M$ particles at the energy $E$ with the boundary condition $\omega$. Using Equations (5.4) and (5.6) one may express the normalization of the measure for $q, p$ in Equation (5.7) by means of $W_{D, \Lambda}(e, n \mid \omega)$. Thus, using $|x|_{+}=\max (0, x)$,

$$
\begin{aligned}
W_{D, \Lambda}(e, n-1 \mid \omega) \geqq & W_{D, \Lambda}(e, n \mid \omega) \\
& \cdot\left\langle(M+1)^{-1} \int_{q \in D} d^{d} \bar{q} \int d^{d} \bar{p}\left|\frac{p^{2}-\bar{p}^{2}-\phi(\bar{q} \mid q)}{p^{2}}\right|_{+}^{d M / 2-1}\right\rangle_{M, E, \omega} \\
\geqq & W_{D, \Lambda}(e, n \mid \omega)\left\langle(M+1)^{-1} \int_{q \in D} d^{d} \bar{q}|p|^{d}\right. \\
& \left.\cdot\left|1-\frac{\phi(\bar{q} \mid q)}{p^{2}}\right|_{+}^{d(M+1) / 2-1} \int d^{d} x\left|1-x^{2}\right|^{d M / 2-1}\right\rangle_{M, E, \omega} .
\end{aligned}
$$

Let $k, \gamma>0$. Using (twice) the inequality

$$
1-z \geqq e^{-2 z} \quad \forall z \in[0,1 / 2]
$$

we obtain, for $M \geqq \max (2 \gamma / k, 2)$

$$
\begin{aligned}
& W_{D, \Lambda}(e, n-1 \mid \omega) \geqq W_{D, \Lambda}(e, n \mid \omega) \bar{c}_{d} k^{d / 2} e^{-2 \gamma d / k} \\
& \cdot\left\langle I\left[p^{2} \geqq k M\right](M+1)^{-1} \int_{D} d^{d} \bar{q} I\left[\phi\left(\bar{q} \mid q, \omega_{D^{c}}\right)<\gamma\right]\right\rangle_{\mu, E, \omega} .
\end{aligned}
$$


(We remind the reader that $I[C]$ is the characteristic function of the set on which the condition $c$ is satisfied.)

Let

$$
Y_{D}(\omega)=I\left[\Sigma_{p^{2}}\left(\omega_{D}\right)>k N_{D}(\omega)\right]\left(N_{D}(\omega)+1\right)^{-1} \int_{D} d^{d} \bar{q} I[\phi(\bar{q} \mid \omega)<\gamma]
$$

and

$$
\mathscr{Y}_{D}(\omega)=\int \mu\left(d \bar{\omega} \mid \mathscr{B}_{\bar{\Lambda}} G_{\overline{\bar{A}}}\right)(\omega) Y_{D}(\bar{\omega}) .
$$

If $N_{D}(\omega) \geqq \max (2 \gamma / k, 2)$ substituting $n=N_{\Lambda}(\omega) e=H_{\Lambda}(\omega)$ in Equation (5.8) leads to

$W_{D, \Lambda}\left(H_{\Lambda}(\omega), N_{\Lambda}(\omega)-1 \mid \omega\right) \geqq W_{D, \Lambda}\left(H_{\Lambda}(\omega), N_{\Lambda}(\omega) \mid \omega\right) c(d, \gamma, k) \mathscr{Y}_{\Lambda}(\omega)$.

The functions $g_{\gamma}(\omega)$ [Eq. (5.1)] and $\hat{p}(\omega)$ [Eq. (5.2)] are measurable with respect to $G_{\infty}$ and therefore constant a.e. for the extremal C.E. state $\mu$. Therefore $\exists \gamma, k, \delta>0$ such that $\forall \Lambda, \bar{\Lambda}, \Lambda \subset \bar{\Lambda}$,

$$
\begin{aligned}
& I\left[\Sigma_{p^{2}}\left(\omega_{D}\right)>k N_{D}(\omega)\right] \underset{\overline{\bar{A} \uparrow \mathbb{R}^{d}}}{\longrightarrow} 1 \bmod \mu \\
& \lim _{\overline{\bar{\Lambda} \uparrow \mathbb{R}^{d}}} \inf \left(N_{D}(\omega)+1\right)^{-1} \int_{D} d^{d} \bar{q} I\left[\phi\left(\bar{q} \mid q, \omega_{D^{c}}\right)<\gamma\right] \geqq 2 \delta \bmod \mu .
\end{aligned}
$$

Thus, for this choice of $k$ and $\gamma$

$$
\lim _{\overline{\bar{\Lambda}} \uparrow \mathbb{R}^{d}} \inf Y_{D}(\omega) \geqq 2 \delta .
$$

Let

$$
A_{D}=\left\{\omega \mid Y_{D}(\omega)<\delta\right\} .
$$

By Equation (5.9) $I_{A_{D}} \rightarrow 0 \bmod \mu$. Therefore

$$
\int \mu(d \omega) \mu\left(A_{D} \mid \mathscr{B}_{\bar{\Lambda} \backslash \Lambda} G_{\overline{\bar{\Lambda}}}\right)(\omega)=\mu\left(A_{D}\right) \rightarrow 0 \quad \text { as } \quad \overline{\bar{\Lambda}} \uparrow \mathbb{R}^{d} .
$$

Passing to a subsequence we obtain a sequence $\overline{\bar{\Lambda}} \uparrow \mathbb{R}^{d}$ (which depends only on $\Lambda, \bar{\Lambda}$ ) for which the convergence is pointwise:

$$
\lim _{\overline{\bar{\Lambda} \uparrow \mathbb{R}^{d}}} \mu\left(A_{D} \mid \mathscr{B}_{\bar{\Lambda} \backslash \Lambda} G_{\bar{\Lambda}}\right)(\omega)=0 \bmod \mu .
$$

Since

$$
\begin{aligned}
& \mathscr{Y}_{D}(\omega) \geqq \delta \mu\left(A_{D}^{c} \mid \mathscr{B}_{\bar{\Lambda} \backslash \Lambda} G_{\overline{\bar{A}}}\right)(\omega)=\delta\left(1-\mu\left(A_{D} \mid \mathscr{B}_{\bar{\Lambda} \backslash \Lambda} G_{\overline{\bar{A}}}\right)(\omega)\right) \\
& \lim _{\overline{\bar{A} \uparrow \mathbb{R}^{d}}} \inf \mathscr{Y}_{D}(\omega) \geqq \delta \bmod \mu .
\end{aligned}
$$

Thus

$$
\lim _{\overline{\bar{\Lambda} \uparrow \mathbb{R}^{d}}} \inf \frac{W_{D, \Lambda}\left(H_{\Lambda}(\omega), N_{\Lambda}(\omega)-1 \mid \omega\right)}{W_{D, \Lambda}\left(H_{\Lambda}(\omega), N_{\Lambda}(\omega) \mid \omega\right)} \geqq c(d, \gamma, k) \delta>0 \bmod \mu,
$$

proving Claim 1ii).

Let

$$
e_{\Lambda}^{0}(n, \omega)=\inf \left\{H_{\Lambda}(\bar{\omega}) \mid \bar{\omega}_{A^{c}}=\omega_{\Lambda^{c}}, N_{\Lambda}(\bar{\omega})=n\right\} .
$$


Since $\mu\left(\cdot \mid G_{A}\right)(\omega)$ are given by the microcanonical ensembles, $\forall, \bar{\Lambda}$

$$
\mu\left\{\omega \mid \omega_{\bar{A}} \ni\{(q, p),(\bar{q}, \bar{p})\} \text { such that } p=0, \bar{p} \neq 0\right\}=0 .
$$

Taking the limit $\bar{\Lambda} \uparrow \mathbb{R}^{d}$ and using the fact that by Equation (5.2), $\mu$-a.e. $\omega$ includes a particle with positive kinetic energy, we may conclude that the kinetic energy of any particle in $\omega$ does not vanish $(\bmod \mu)$. Thus

$$
N_{\Lambda}(\omega)>0 \text { implies } H_{\Lambda}(\omega)>e_{\Lambda}^{0}\left(N_{\Lambda}(\omega), \omega\right) \bmod \mu \text {. }
$$

In fact since, by Equation (5.3) the total kinetic energy is infinite, for $\mu$-a.e. $\omega$

$$
H_{\Lambda}(\omega)>e_{\Lambda}^{0}\left(N_{\Lambda}(\omega)-1, \omega\right)
$$

for all $\Lambda$ large enough. (Here the finiteness of the range of the potential was used.)

We shall now prove the following claim, from which the hypotheses of Lemma 5.1 follow directly.

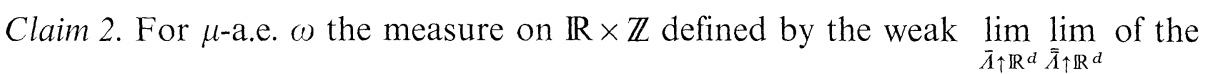
distributions of $\left(H_{\Lambda}(\bar{\omega}), N_{\Lambda}(\bar{\omega})\right)$ in the states

$$
\mu\left(d \bar{\omega} \mid \mathscr{B}_{\bar{\Lambda} \backslash \Lambda} G_{\overline{\bar{A}}}\right)(\omega)\left(\rightarrow \mu\left(\cdot \mid \bar{L}_{\Lambda}\right)(\omega)\right)
$$

is i) absolutely continuous on $\mathbb{R} \times\{n>0\}$,

ii) equivalent to the Lebesgue measure on

$$
\left[e_{\Lambda}^{0}\left(N_{\Lambda}(\omega), \omega\right), H_{\Lambda}(\omega)\right] \times\left\{N_{\Lambda}(\omega)\right\},
$$

iii) equivalent to the Lebesgue measure on

$$
\left[e_{\Lambda}^{0}\left(N_{\Lambda}(\omega)-1, \omega\right), H_{\Lambda}(\omega)\right] \times\left\{N_{\Lambda}(\omega)-1\right\}
$$

for $\Lambda$ such that Equation (5.11) is satisfied.

Let $n>0$. With respect to $\mu\left(d \bar{\omega} \mid \mathscr{B}_{\bar{\Lambda} \backslash \Lambda} G_{\bar{\Lambda}}\right)(\omega)$ the distribution of $H_{\Lambda}(\bar{\omega})$ for $N_{\Lambda}(\bar{\omega})=n$ is expressed in Equation (5.5) as a product of $R_{\Lambda}(e, n \mid \omega)$, whose support is $\left[e_{\Lambda}^{0}(n, \omega), \infty\right)$, with monotone decreasing functions. This implies that the measures obtained by multiplying these distributions by $R_{A}^{-1}(e, n \mid \omega)$ are monotone in the sense that the integrals of positive continuous functions with compact support in $\left(e_{A}^{0}(n, \omega), \infty\right)$ decrease when these functions are translated to the right. This monotonicity is clearly preserved under limits.

Any locally finite measure $\sigma$ on $(e, \infty)$ which is monotone has support in some interval (possibly empty) in which it is equivalent to the Lebesgue measure. To prove this notice that it is implied by the concavity of the shifted distribution functions

$$
F_{y}(x)=\operatorname{sgn}(x-y) \sigma((x, y) \cup(y, x))
$$

which follows from the monotonicity. Therefore, under $\mu\left(\cdot \mid \bar{L}_{A}\right)(\omega)$ the distribution of $H_{A}$ for $N_{A}=n$ is of this form except for a possible additional point measure at $e_{\Lambda}^{0}(n, \omega)$.

The limiting measures $\mu\left(\cdot \mid \bar{L}_{A}\right)(\omega)$ are obtained from $\mu$ by conditioning. Therefore any property holds $\bmod \mu$ if and only if it holds almost surely for the limiting measures for $\mu$-a.e. $\omega$. 
We shall now use this fact to prove each part of the claim.

i) Equation (5.10) implies that for any $n>0$

$\mu\left(\left\{H_{\Lambda}(\cdot)=e_{\Lambda}^{0}(n, \cdot)\right\} \cap\left\{N_{\Lambda}(\cdot)=n\right\} \mid \bar{L}_{\Lambda}\right)(\omega)=0 \bmod \mu$.

Since with respect to these measures $e_{A}^{0}(n, \cdot)=e_{\Lambda}^{0}(n, \omega)$ the above mentioned discrete component of the distribution of $H_{\Lambda}$ vanishes. This proves part i) of Claim 2.

ii) Let $\hat{e}_{\Lambda}(n, \omega)$ be the right end point of the support of the energy distribution on $\left\{N_{A}=n\right\}$ under $\mu\left(\cdot \mid \bar{L}_{A}\right)(\omega)$. Thus for $\mu$-a.e. $\omega$

$$
H_{\Lambda}(\bar{\omega}) \leqq \hat{e}_{\Lambda}\left(N_{\Lambda}(\bar{\omega}), \omega\right)=\hat{e}_{\Lambda}\left(N_{\Lambda}(\bar{\omega}), \bar{\omega}\right)
$$

for $\mu\left(d \bar{\omega} \mid \bar{L}_{A}\right)(\omega)$-a.e. $\bar{\omega}$. I.e.,

$$
H_{\Lambda}(\omega) \leqq \hat{e}_{\Lambda}\left(N_{\Lambda}(\omega), \omega\right) \bmod \mu
$$

proving Part ii) of Claim 2.

iii) From Claim 1ii) and from Claim 2ii) we conclude that, $\bmod \mu, \exists c>0$ and $\overline{\bar{\Lambda}} \uparrow \mathbb{R}^{d}$ such that

$$
\lim _{\overline{\bar{\Lambda} \uparrow \mathbb{R}^{d}}} \inf \frac{W_{D, \Lambda}(e, n-1 \mid \omega)}{W_{D, \Lambda}(e, n \mid \omega)} \geqq c
$$

for a.e. $e \in\left[e_{\Lambda}^{0}(n, \omega), \hat{e}_{\Lambda}(n, \omega)\right]$. Thus

$$
\begin{aligned}
& \mu\left(H_{\Lambda} \in d e, N_{\Lambda}=n-1 \mid L_{\Lambda, \bar{\Lambda}}\right)(\omega) \\
& \quad \geqq c \frac{R_{\Lambda}(e, n-1 \mid \omega)}{R_{\Lambda}(e, n \mid \omega)} \mu\left(H_{\Lambda} \in d e, N_{\Lambda}=n \mid L_{\Lambda, \bar{\Lambda}}\right)(\omega) .
\end{aligned}
$$

Passing to the limit $\bar{\Lambda} \uparrow \mathbb{R}^{d}$ and using Claim 2ii), Part iii) of Claim 2 follows.

As remarked above the hypotheses of Lemma 5.1 follow from Claim 2. Thus Theorem 5.1 is proven.

\section{Canonical Gibbs States}

There are three classical ensembles. We have discussed conditions on states of infinite systems which correspond to two of these. The following condition, which was studied in $[6,8]$, characterizes limits of canonical ensembles. It was this condition which first motivated our interest in C.E. when it was proven $[9,12]$ that it is equivalent to a type of dynamical stability.

Definition 6.1. For a given extensive (energy) function $H$, a state $\mu$ is a canonical Gibbs state of inverse temperature $\beta$ if for any $\Lambda$ there is a Borel measurable function $b_{\Lambda}: \mathbb{Z} \times \Omega \rightarrow \mathbb{R}$ such that for $\mu$-a.e. $\omega$

$$
\mu_{\Lambda}(d \xi \mid \omega)=e^{-\beta H\left(\xi \mid \omega_{\Lambda}\right)+b_{\Lambda}\left(N_{\Lambda}(\xi), \omega\right)} v_{\Lambda}(d \xi) .
$$

Remark 6.1. Clearly, any canonical Gibbs state is also a C.E. state. Notice, however, that an extremal canonical Gibbs state need not be an extremal C.E. state. 
Thus we may conclude from Theorem 5.1 that under the assumptions appropriate a canonical Gibbs state is a convex combination of Gibbs states. In fact, as may easily be seen, all the Gibbs states are of the same temperature.

A more powerful approach results from the observation that canonical Gibbs states may be viewed as the C.E. states with respect to the single extensive quantity $N$ for the modified a priori measure obtained by replacing $v_{\Lambda}(d \xi)$ by $\exp \left[-\beta H\left(\xi \mid \omega_{\Lambda^{c}}\right)\right] v_{\Lambda}(d \xi)$.

The only property of $v$ which was used is that it induces on the entropy function the "quasi-extensiveness" expressed in Lemma 3.1. Since the above modification of the a priori measure increases the entropy function by an extensive quantity, Lemma 3.1 still holds.

Thus the arguments of Section 4 apply, and in fact the details simplify greatly since in this case $U=\mathbb{Z}$. The appropriate notion of looseness is that $\exists \Lambda$ and $n \in \mathbb{Z}$ such that

$$
\mu\left\{\omega \mid \mu_{\Lambda}\left(N_{\Lambda}=n \mid \omega\right) \neq 0, \mu\left(N_{\Lambda}=n+1 \mid \omega\right) \neq 0\right\}>0 .
$$

With this notion we obtain in place of Theorem 4.3 :

Theorem 6.1. Every loose extremal canonical Gibbs state is a Gibbs state.

In place of Theorem 5.1 we obtain the following result which no longer requires the presence of momentum degrees of freedom.

Theorem 6.2. For a finite range interaction, every unsaturated canonical Gibbs state with an infinite number of particles is a convex combination of Gibbs states.

$(N=\infty$ automatically in C.E. states for translation invariant potentials.)

To prove this one need only establish looseness which in this case follows by a greatly simplified version of the argument in Section 5. Let

$$
Z_{\Lambda}\left(n, \omega_{\Lambda^{c}}\right)=\int_{N_{\Lambda}(\xi)=n} v_{\Lambda}(d \xi) e^{-\beta H\left(\xi \mid \omega_{\Lambda} c\right)} .
$$

The basic required estimates are some positive lower bounds for

$$
Z_{\Lambda}\left(N_{\Lambda}(\omega)+1, \omega_{\Lambda^{c}}\right) / Z_{\Lambda}\left(N_{\Lambda}(\omega), \omega_{\Lambda^{c}}\right)=\left\langle\left[N_{\Lambda}(\omega)+1\right]^{-1} \int_{\Lambda} d q e^{-\beta \phi(q \mid \omega)}\right\rangle_{\Lambda, \omega}^{(\text {can. })} .
$$

where \langle\rangle$_{\Lambda, \omega}^{(\text {can.) }}$ is the expectation in the canonical ensemble in $\Lambda$ with $N_{\Lambda}(\omega)$ particles and the boundary conditions $\omega_{A^{c}}$.

To establish looseness appropriate upper bounds would do as well. Thus one proves

Theorem 6.3. For a finite range interaction coming from potentials which are either

1) positive or

2) hard core and bounded below

every canonical Gibbs state with a positive density is a convex combination of Gibbs states.

Acknowledgements. M. Aizenman is very grateful to E. Lieb for stimulating discussions. He wishes also to thank J. Barnes for gracious hospitality at the Los Alamos Scientific Laboratory where part of this work was done. S. Goldstein wishes gratefully to acknowledge the Institute for Advanced Studies of Hebrew University for providing the opportunity to pursue part of this work. J. Lebowitz would like to acknowledge useful discussion with H. Georgii, O. Lanford, C. Gruber, and P. Martin. 


\section{References}

1. Ruelle, D.: Statistical mechanics. New York: Benjamin 1969

2. Lanford III,O.E., Ruelle, D. : Commun. math. Phys. 13, 194 (1969)

3. Dobrushin, R.L. : Funct. Anal. Appl. 2, 31 (1968)

4. Bogoliubov, N. N.: J. Phys. USSR 10, 257, 265 (1946)

5. Thompson, R. L.: Mem. A. M. S. 150 (1974)

6. Georgii,H.: Z. Wahrscheinlichkeitstheorie verw. Gebiete 32, 272 (1975); 33, 331 (1976)

7. Martin-Löf, A. : The equivalence of ensembles and Gibbs phase rule for classical lattice systems: J. Stat. Phys. (to appear)

8. Georgii,H.: Commun. math. Phys. 48, 37 (1976)

9. Aizenman, M., Gallavotti,G., Goldstein,S., Lebowitz,J.L. : Commun. math. Phys. 48, 1 (1976)

10. Haag, R., Kastler, D., Trych-Pohlmeyer, E.: Commun. math. Phys. 38, 173 (1974)

11. Gallavotti,G., Verboven,E.: Nuovo Cimento 28B, 274 (1975)

12. Aizenman,M., Goldstein,S., Gruber,C., Lebowitz,J.L., Martin,P.: Commun. math. Phys. 53, 209 (1977)

13. Preston, C.J.: Canonical and microcanonical Gibbs states. Preprint (1977)

14. Schwartz, L.: Radon measures. London, New York: Oxford University Press (1973)

15. Lanford III, O.E.: Time evolution of large classical systems. In: Dynamical systems, theory, and applications. In: Lecture notes in physics, Vol. 38. Berlin. Heidelberg, New York: Springer 1975

16. Föllmer, H.: In: Seminaire de probabilites IX. Lecture Notes in Mathematics, Vol. 465. Berlin, Heidelberg, New York: Springer 1975

17. Dynkin,E.B.: Actes, Congrès intern. Math., 1970, Tome 2, 507 (1971)

Communicated by J. Glimm

Received May 18, 1978 